\title{
On the chromatic number of random graphs with a fixed degree sequence
}

\author{
Alan Frieze* $\quad$ Michael Krivelevich ${ }^{\dagger} \quad$ Cliff Smyth ${ }^{\ddagger}$
}

December 13, 2006

\begin{abstract}
Let $\mathbf{d}=1 \leq d_{1} \leq d_{2} \leq \cdots \leq d_{n}$ be a non-decreasing sequence of $n$ positive integers, whose sum is even. Let $\mathcal{G}_{n, \mathbf{d}}$ denote the set of graphs with vertex set $[n]=$ $\{1,2, \ldots, n\}$ in which the degree of vertex $i$ is $d_{i}$. Let $G_{n, \mathbf{d}}$ be chosen uniformly at random from $\mathcal{G}_{n, \mathbf{d}}$. Let $d=\left(d_{1}+d_{2}+\cdots+d_{n}\right) / n$ be the average degree. We give a condition on $\mathbf{d}$ under which we can show that whp the chromatic number of $\mathcal{G}_{n, \mathbf{d}}$ is $\Theta(d / \ln d)$. This condition is satisfied by graphs with exponential tails as well those with power law tails.
\end{abstract}

\section{Introduction}

Let $\mathbf{d}=1 \leq d_{1} \leq d_{2} \leq \cdots \leq d_{n}$ be a fixed non-decreasing sequence of $n$ positive integers, whose sum is even. Let $\mathcal{G}_{n, \mathbf{d}}$ denote the set of graphs with vertex set $[n]=\{1,2, \ldots, n\}$ in which the degree of vertex $i$ is $d_{i}$. Let $G_{n, \mathbf{d}}$ be chosen uniformly at random from $\mathcal{G}_{n, \mathbf{d}}$. When $d_{i}=r$ for $i \in[n]$ then this models a random $r$-regular graph $G_{n, r}$ and there is a large literature on this subject. We refer the reader to the survey by Wormald [18] for an excellent summary. By now we know much about the structure of random regular graphs.

For general $\mathbf{d}$, less is known. In many, but not all, cases we can estimate $\left|\mathcal{G}_{n, \mathbf{d}}\right|$. See Bender and Canfield [5], McKay and Wormald [13, 14]. We have the configuration model to study them, Bollobás [6]. We know something of their connectivity properties, Molloy and Reed

*Department of Mathematical Sciences, Carnegie Mellon University, Pittsburgh PA15213, U.S.A. Supported in part by NSF grant CCR-0200945.

${ }^{\dagger}$ Department of Mathematics, Raymond and Beverly Sackler Faculty of Exact Sciences, Tel Aviv University, Tel Aviv 69978, Israel. E-mail: krivelev@post.tau.ac.il. Research supported in part by USA-Israel BSF Grant 2002-133 and by grants 64/01 and 526/05 from the Israel Science Foundation.

‡Department of Mathematics, Massachusetts Institute of Technology, Cambridge, MA 02139, U.S.A. 
$[16,17]$.(See also Cooper and Frieze [8] for the connectivity properties of random digraphs with a fixed degree sequence). They have been used in the context of massive graph models of telephone networks and the WWW, Aiello, Chung and Lu [3].

In this paper we will concern ourselves with the chromatic number of $G_{n, \mathbf{d}}$. What should we expect? For $r$-regular graphs it is known that when $r$ is large $\chi\left(G_{n, r}\right) \sim \frac{r}{2 \ln r} \mathbf{w h p}^{1}$, see Frieze and Euczak [10] and Achlioptas and Moore [1] (see also Cooper et al. [9] for extension of some of these results to the case where $r=r(n) \leq n^{1-\eta}$, for an arbitrary small constant $\eta>0)$. For the random graph $G_{n, p}$ where the average degree $d=(n-1) p$ is large, it is known that $\chi\left(G_{n, p}\right) \sim \frac{d}{2 \ln d}$, see Bollobás [7], Euczak [12] and Achlioptas and Naor [2]. So if we let

$$
d=\frac{d_{1}+d_{2}+\cdots+d_{n}}{n}
$$

be the average degree in $G_{n, \mathbf{d}}$ then we might hope to prove that $\chi\left(G_{n, \mathbf{d}}\right) \sim \frac{d}{2 \ln d} \mathbf{w h} \mathbf{p}$. This is too much to expect given the variety of possible degree sequences. Indeed if $\nu=\frac{n}{\ln n}$ and

$$
d_{i}= \begin{cases}1 & i \leq n-\nu \\ d \ln n & i>n-\nu\end{cases}
$$

then the average degree $\sim d$, but whp the sub-graph $H$ induced by the $\nu$ largest degree vertices has average degree close to $d \ln n$ when $d$ is large. Then whp $\chi(H) \sim \frac{d \ln n}{2 \ln \ln n}$ for $\ln d=o(\ln \ln n)$ and so we will have to be less ambitious in our goals ${ }^{2}$. Let

$$
D_{k}=d_{n}+d_{n-1}+\cdots+d_{n-k+1}
$$

be the sum of the $k$ largest degrees.

Let

$$
M_{1}=D_{n}=d n \text { and } M_{2}=\sum_{i=1}^{n} d_{i}\left(d_{i}-1\right) \leq \Delta M_{1} \text { where } \Delta=d_{n}
$$

\section{Theorem 1}

1. Suppose that there exist constants $1 / 2<\alpha<1, \epsilon, K_{0}>0$ and $\omega=\omega(n) \rightarrow \infty$ such that

(a)

$$
D_{k} \leq K_{0} d n(k / n)^{\alpha}
$$

for $k \leq \epsilon n$.

(b) $\Delta^{5} \leq M_{2} / \omega$.

\footnotetext{
${ }^{1}$ A sequence of events $\mathcal{E}_{n}$ occurs with high probability (whp) if $\lim _{n \rightarrow \infty} \operatorname{Pr}\left(\mathcal{E}_{n}\right)=1$.

${ }^{2}$ The methods of [9] can be used to verify this claim.
} 
Then there exists $b_{1}$ dependent only on $\alpha, \epsilon, K_{0}$ such that whp

$$
\chi\left(G_{n, \mathbf{d}}\right) \leq b_{1} \frac{d}{\ln d} .
$$

2. Suppose only that $\Delta^{4} \leq M_{1} / \omega$ (a weaker condition than 1(b)), then there exists $b_{2}$ such that whp

$$
\chi\left(G_{n, \mathbf{d}}\right) \geq b_{2} \frac{d}{\ln d} .
$$

Notice that if Condition (a) of the theorem holds for $\alpha, \epsilon, K_{0}$ then it also holds when $K_{0}$ is replaced by a larger constant. As we increase $K_{0}$ our bound $b_{1}$ will decrease. We are therefore justified in assuming throughout that $K_{0}$ is sufficiently large that some inequalities are valid.

Condition (b) is chosen so that we can use the results of [15]. It may be possible to prove our results under the less stringent conditions of [14], but there are difficulties, as will be pointed to later.

A referee has suggested that the quantity $d^{\prime}=\max _{k} D_{k}^{2} / d n k$ (which can be viewed as the probabilistic version of the "maximum average degree") might be a better predictor of chromatic number. This could well be true and it makes for an interesting research question. We also suspect that if $d_{i} \sim d f(i / n)$ where $f$ is some "nice" real valued function on $[0,1]$ such that $\int_{0}^{1} f(x) d x=1$ then we might be able to determine the chromatic number of $G_{n, \mathbf{d}}$ asymptotically.

The proof of the upper bound is given in Section 3. The proof of the lower bound is given in Section 4. We have made no attempt to optimize constants.

\section{Degree sequences that satisfy (1)}

It is natural to ask whether there many types of degree sequence that satisfy the conditions of the first part of the theorem.

We first consider a degree sequence with a power law tail. For integer $\ell \geq 1$ we let $\nu_{\ell}$ denote the number of vertices of degree $\ell$. Our assumption is that there are some constants $A>0$ and $\gamma>3$ such that for $\ell \geq(A / \epsilon)^{1 /(\gamma-1)}$

$$
\nu_{\ell} \leq \begin{cases}0 & \ell \leq 1 \\ \left\lfloor A d \ell^{-\gamma} n\right\rfloor & 2 \leq \ell \leq n^{1 / 5} / \ln n \\ 0 & \ell>n^{1 / 5} / \ln n\end{cases}
$$

Here we have $\alpha=\frac{\gamma-2}{\gamma-1}>1 / 2$. 
We have forced Condition (b) and so we only need to check Condition (a). Suppose now that $k=\kappa n \leq \epsilon n$. Then

$$
D_{k} \leq A d n \sum_{\ell \geq r} \ell^{-(\gamma-1)}=\left(1+\delta_{r}\right) \frac{A d n}{\gamma-2} r^{2-\gamma}
$$

where $\delta_{r} \rightarrow 0$ as $r \rightarrow \infty$ and

$$
r=\min \left\{\rho: A d \sum_{\ell \geq \rho} \ell^{-\gamma} \geq \kappa\right\}=\left(1+\delta_{\kappa}^{\prime}\right)\left(\frac{A d}{(\gamma-1) \kappa}\right)^{1 /(\gamma-1)}
$$

where $\delta_{\kappa}^{\prime} \rightarrow 0$ as $\kappa \rightarrow 0$. Thus,

$$
D_{k} \leq\left(1+\delta_{r}\right)\left(1+\delta_{\kappa}^{\prime}\right)^{2-\gamma}(\gamma-2)^{-1} A\left(\frac{\gamma-1}{A d}\right)^{\alpha} d n \kappa^{\alpha}
$$

and we have the condition of Theorem 1.

A similar argument holds if we assume that our degree sequence has an exponential tail viz. for some constants $A>0$ and $0<\epsilon \ll \gamma<1$ we have for $\ell \geq\left\lfloor\ln _{1 / \gamma}(A d / \epsilon)\right\rfloor$

$$
\nu_{\ell} \leq A d \gamma^{\ell} n
$$

Note that whp the degree sequence of $G_{n, p}, p=c / n, c$ constant, satisfies this condition.

Suppose now that $k=\kappa n \leq \epsilon n$. Then

$$
D_{k} \leq A d n \sum_{\ell \geq r} \ell \gamma^{\ell} \leq A d n \int_{x=r-1}^{\infty} x \gamma^{x} d x=\frac{A d n \gamma^{r-1}}{\ln 1 / \gamma}\left(r-1+\frac{1}{\ln 1 / \gamma}\right)
$$

where

$$
r=\min \left\{\rho: A d \sum_{\ell \geq \rho} \ell \gamma^{\ell} \geq \kappa\right\} \sim \ln _{1 / \gamma}\left(\frac{A d}{\kappa \ln 1 / \gamma}\right)
$$

as $\epsilon \rightarrow 0$.

Thus,

$$
D_{k} \leq 2 \kappa n
$$

and we have the condition of Theorem 1 with room to spare.

\section{$3 \quad$ Upper bound}

\subsection{Configurations}

We will work initially in the configuration model and then show how our results can be justified in the uniform model. Let $W=[n d]$ be our set of points and let $W_{i}=\left[d_{1}+\right.$ 
$\left.\cdots+d_{i-1}+1, d_{1}+\cdots+d_{i}\right], i \in[n]$, partition $W$. The function $\phi: W \rightarrow[n]$ is defined by $w \in W_{\phi(w)}$. Given a pairing $F$ (i.e. a partition of $W$ into $m=d n / 2$ pairs) we obtain a (multi-)graph $G_{F}$ with vertex set $[n]$ and an edge $(\phi(u), \phi(v))$ for each $\{u, v\} \in F$. Choosing a pairing $F$ uniformly at random from among all possible pairings of the points of $W$ produces a random (multi-)graph $G_{F}$.

This model is valuable because of the following easily proven fact: Suppose $G \in \mathcal{G}_{n, \mathbf{d}}$. Then

$$
\operatorname{Pr}\left(G_{F}=G \mid G_{F} \text { is simple }\right)=\frac{1}{\left|\mathcal{G}_{n, \mathbf{d}}\right|} .
$$

It follows that if $G$ is chosen randomly from $\mathcal{G}_{n, \mathbf{d}}$, then for any graph property $\mathcal{P}$

$$
\operatorname{Pr}(G \in \mathcal{P}) \leq \frac{\operatorname{Pr}\left(G_{F} \in \mathcal{P}\right)}{\operatorname{Pr}\left(G_{F} \text { is simple }\right)}
$$

Before starting the proof of the upper bound in Theorem 1, we introduce some notation that we will use throughout the course of the proof. Let

$$
\theta=\max \left\{d^{2 /(2 \alpha+1)}, \frac{16}{2 \alpha-1}\right\}
$$

and

$$
\epsilon_{1}=\min \left\{\epsilon,\left(\frac{(2 \alpha-1) \theta}{K_{1} d}\right)^{2 /(2 \alpha-1)}\right\} .
$$

for some sufficiently large $K_{1}$.

For a set $U \subseteq V\left(G_{F}\right)$ we denote by $D(U)$ the sum of the degrees of $U$ in $G_{F}$.

We first prove

Lemma 1 Whp $G_{F}$ does not contain a set of vertices $S$ with $k=|S| \leq \epsilon_{1} n$, such that $S$ induces a sub-graph $G[S]$ with minimum degree at least $\theta$.

Proof Suppose that there exists a vertex set $S$ of size $k \in\left[\theta, \epsilon_{1} n\right]$ that induces a graph $G[S]$ with minimum degree at least $\theta$. Let $S_{1}$ be the $k / 2$ lowest degree vertices of $S$ and let $S_{2}=S \backslash S_{1}$. For vertex sets $S, T$ let $e(S)$ denote the number of edges contained in $S$ and let $e(S: T)$ denote the number of edges joining $S$ and $T$.

At least one of the following two events must occur:

$$
\begin{aligned}
& \mathcal{E}_{1}: e\left(S_{1}: S_{2}\right) \geq \theta k / 4 \\
& \mathcal{E}_{2}: e\left(S_{1}\right) \geq \theta k / 8
\end{aligned}
$$


This follows because

$$
2 e\left(S_{1}\right)+e\left(S_{1}: S_{2}\right)=\sum_{v \in S_{1}} d_{G[S]}(v) \geq \theta k / 2
$$

Now $k \leq \epsilon_{1} n$ and so,

$$
d n-\theta k / 2 \geq d n-\theta \epsilon_{1} n / 2 \geq \frac{99}{100} d n
$$

provided

$$
K_{1} \geq 16\left(\frac{1600}{2 \alpha-1}\right)^{\alpha-1 / 2}
$$

(We have chosen $K_{1}$ large enough so that whichever of $d^{2 /(2 \alpha+1)}, \frac{16}{2 \alpha-1}$ is the larger, $\theta \epsilon_{1}<$ 1/100.)

And so

$$
\begin{aligned}
\operatorname{Pr}\left(\mathcal{E}_{1}\right) & \leq \sum_{k=\theta}^{\epsilon_{1} n} \sum_{|S|=k}\left(\begin{array}{c}
D\left(S_{1}\right) \\
\theta k / 4
\end{array}\right)\left(\frac{D\left(S_{2}\right)}{d n-\theta k / 2}\right)^{\theta k / 4} \\
& \leq \sum_{k=\theta}^{\epsilon_{1} n}\left(\begin{array}{c}
n \\
k
\end{array}\right)\left(\begin{array}{c}
D_{k / 2} \\
\theta k / 4
\end{array}\right)\left(\frac{D_{k / 2}}{d n-\theta k / 2}\right)^{\theta k / 4} \\
& \leq \sum_{k=\theta}^{\epsilon_{1} n}\left(\frac{e n}{k}\right)^{k}\left(\frac{5 e D_{k / 2}^{2}}{\theta k d n}\right)^{\theta k / 4} \\
& \leq \sum_{k=\theta}^{\epsilon_{1} n}\left(\frac{e n}{k}\right)^{k}\left(\frac{5 e K_{0}^{2} d}{2^{2 \alpha} \theta} \cdot\left(\frac{k}{n}\right)^{2 \alpha-1}\right)^{\theta k / 4} \\
& \leq \sum_{k=\theta}^{\epsilon_{1} n}\left(\left(\frac{k}{n}\right)^{(2 \alpha-1) \theta / 4-1}\left(\frac{5 e^{2} K_{0}^{2} d}{2^{2 \alpha} \theta}\right)^{\theta / 4}\right)^{k} \\
& \leq \sum_{k=\theta}^{\epsilon_{1} n}\left(\left(\frac{k}{n}\right)^{3(2 \alpha-1) \theta / 16}\left(\frac{5 e^{2} K_{0}^{2} d}{2^{2 \alpha} \theta}\right)^{\theta / 4}\right)^{k} \\
& \leq \sum_{k=\theta}^{\epsilon_{1} n}\left(\left(\frac{k}{n}\right)^{(2 \alpha-1) / 4} \frac{5 e^{2} K_{0}^{2}}{2^{2 \alpha}}\right)^{\theta k / 4} \\
& =o(1),
\end{aligned}
$$

if we choose $K_{1} \geq 100 e^{4} K_{0}^{4}$ (we used the assumption $k / n \leq \epsilon_{1} \leq\left((2 \alpha-1) \theta /\left(K_{1} d\right)\right)^{2 /(2 \alpha-1)} \leq$ $\left(16 / K_{1}\right)^{2 /(2 \alpha-1)}$ in the last line).

Explanation of (4): At least $\theta k / 4$ of the points corresponding to vertices of $S_{1}$ are to be paired with points corresponding to vertices of $S_{2}$. When pairing points, the probability of pairing with a point corresponding to $S_{2}$ is never more than $\frac{D\left(S_{2}\right)}{d n-\ell}$, given up to $\ell / 2-1$ previous pairings. 
In the following estimate, $\ell=\ell\left(S_{1}\right)$ is the total degree of the subgraph $G\left[S_{1}\right]$. Here $\theta k / 4 \leq \ell \leq K_{0} \epsilon_{1}^{\alpha} d n$. Now the maximum degree in $S_{1}$ is at most $\frac{D_{k / 2}}{k / 2} \leq K_{0} d(2 n / k)^{1-\alpha}$ and so

$$
\begin{aligned}
& \operatorname{Pr}\left(\mathcal{E}_{2}\right) \\
& \leq \sum_{k=\theta}^{\epsilon_{1} n} \sum_{S_{1}=\left\{i_{1}<\cdots<i_{k / 2}\right\}} \sum_{\ell=\theta k / 4}^{K_{0} \epsilon_{1}^{\alpha} d n} \sum_{a_{1}+\cdots+a_{k / 2}=\ell} \frac{\left(\begin{array}{c}
k / 2 \\
2 \\
\ell / 2
\end{array}\right)}{(d n-\ell)^{\ell / 2}} \prod_{t=1}^{k / 2} d_{i_{t}}^{a_{t}}
\end{aligned}
$$

$a_{1}, a_{2}, \ldots a_{k / 2}$ is the degree sequence of $G\left[S_{1}\right]$ (more explanation below)

$$
\begin{aligned}
& \leq \sum_{k=\theta}^{\epsilon_{1} n} \sum_{S_{1}=\left\{i_{1}<\cdots<i_{k / 2}\right\}} \sum_{\ell=\theta k / 4}^{K_{0} \epsilon_{1}^{\alpha} d n} \sum_{a_{1}+\cdots+a_{k / 2}=\ell}\left(\frac{k^{2}}{\ell d n}\right)^{\ell / 2} \prod_{t=1}^{k / 2}\left(K_{0} d(2 n / k)^{(1-\alpha)}\right)^{a_{t}} \\
& \leq \sum_{k=\theta}^{\epsilon_{1} n} \sum_{S_{1}=\left\{i_{1}<\cdots<i_{k / 2}\right\}} \sum_{\ell=\theta k / 4}^{K_{0} \epsilon_{1}^{\alpha} d n} \sum_{a_{1}+\cdots+a_{k / 2}=\ell}\left(\frac{16 K_{0}^{2} d k^{2 \alpha-1}}{\theta n^{2 \alpha-1}}\right)^{\ell / 2} \\
& \leq \sum_{k=\theta}^{\epsilon_{1} n} \sum_{S_{1}=\left\{i_{1}<\cdots<i_{k / 2}\right\}} \sum_{\ell=\theta k / 4}^{K_{0} \epsilon_{1}^{\alpha} d n}\left(\frac{64 K_{0}^{2} d k^{2 \alpha-1}}{\theta n^{2 \alpha-1}}\right)^{\ell / 2} \\
& \leq d n \sum_{k=\theta}^{\epsilon_{1} n}\left(\begin{array}{c}
n \\
k / 2
\end{array}\right)\left(\frac{64 K_{0}^{2} d k^{2 \alpha-1}}{\theta n^{2 \alpha-1}}\right)^{\theta k / 8} \\
& \leq d n \sum_{k=\theta}^{\epsilon_{1} n}\left(\frac{2 e n}{k}\left(\frac{64 K_{0}^{2} d k^{2 \alpha-1}}{\theta n^{2 \alpha-1}}\right)^{\theta / 4}\right)^{k / 2} \\
& \leq d n \sum_{k=\theta}^{\epsilon_{1} n}\left(\frac{2^{12} K_{0}^{4} d^{2} k^{2 \alpha-1}}{\theta^{2} n^{2 \alpha-1}}\right)^{\theta k / 16} \\
& =o(1) .
\end{aligned}
$$

Here we choose $K_{1} \geq 2^{10} K_{0}^{2}$ so that $2^{12} K_{0}^{4} d^{2} \epsilon_{1}^{2 \alpha-1}<\theta^{2} / 2$.

Explanation of (5): Fix $S_{1}=\left\{i_{1}<\cdots<i_{k / 2}\right\}$. Now fix the degrees $a_{1}, \ldots, a_{k / 2}$ of $i_{1}, \ldots, i_{k / 2}$. $\left(\begin{array}{c}\left(\begin{array}{c}k / 2 \\ 2\end{array}\right) \\ \ell / 2\end{array}\right)$ is a crude upper bound on the number of graphs with this set of degrees. Then we use the fact that the probability an edge exists between two vertices of degrees $d_{r}, d_{s}$ is never more than $\frac{d_{r} d_{s}}{d n-\ell}$, given up to $\ell / 2-1$ previous pairings.

Now let

$$
t_{1}=1+\left\lceil\log _{3}\left(2 / \epsilon_{1}\right)\right\rceil
$$

and

$$
I_{t}=\left\{n-\frac{n}{3^{t}}+1, \ldots, n-\frac{n}{3^{t+1}}\right\} \text { for } t=0,1, \ldots, t_{1}
$$


Let $G_{t}$ be the sub-graph of $G_{F}$ induced by $I_{t}$ and for $k \in I_{t}$ let $\tilde{d}_{k}$ be the degree of $k$ in $G_{t}$. Let

$$
\Delta_{t}=\frac{K_{2} d}{2^{(2 \alpha-1) t}}
$$

where $K_{2}=3 \epsilon^{-1} 2^{(2 \alpha-1) t_{0}}+K_{2}^{\prime}+K_{2}^{\prime \prime}$ where

$$
t_{0}=\left\lfloor\log _{3} 1 / \epsilon\right\rfloor
$$

and $K_{2}^{\prime}, K_{2}^{\prime \prime}$ are defined below.

For $0 \leq t \leq t_{1}$ let $B_{t}=\left\{k \in I_{t}: \tilde{d}_{k} \geq \Delta_{t}\right\}, Z_{t}=\left|B_{t}\right|$ and $Z=Z_{0}+\cdots+Z_{t_{1}}$.

Lemma $2 Z \leq \epsilon_{1} n / 2$ whp.

Proof If $k \in I_{t}, t \leq t_{0}$, then there are at least $n-\left|\bigcup_{i=1}^{t} I_{i}\right| \geq 3 \epsilon n$ vertices of degree at least $d_{k}$, and thus $d_{k} \leq 3 \epsilon^{-1} d$ and so $B_{t}=\emptyset, 0 \leq t \leq t_{0}$. For $t>t_{0}$ and $k \in I_{t}$ we see that $\tilde{d}_{k}$ is stochastically dominated by the binomial $B\left(d_{k}, \frac{D_{n / 3^{t}}}{d n}\right)$. Furthermore, since $k \geq n-\epsilon n$ we have

$$
d_{k} \leq \frac{D_{n / 3^{t+1}}}{n / 3^{t+1}} \leq K_{0} 3^{(t+1)(1-\alpha)} d
$$

and

$$
\frac{D_{n / 3^{t}}}{d n} \leq K_{0} 3^{-\alpha t}
$$

So, if $K_{2}^{\prime}=K_{0}^{2} e^{2} 3^{1-\alpha}$ then

$$
\operatorname{Pr}\left(\tilde{d}_{k} \geq \Delta_{t}\right) \leq\left(\frac{K_{0}^{2} 3^{1-\alpha} e}{K_{2}^{\prime}(3 / 2)^{(2 \alpha-1) t}}\right)^{\Delta_{t}} \leq e^{-\Delta_{t}}
$$

(We used: $\operatorname{Pr}[B(n, p) \geq k] \leq\left(\begin{array}{l}n \\ k\end{array}\right) p^{k} \leq\left(\frac{e n p}{k}\right)^{k}$.)

Thus,

$$
\mathbf{E}(Z) \leq n \sum_{t=1}^{t_{1}} 3^{-t} e^{-\Delta_{t}}<n e^{-\Delta_{t_{1}}}
$$

Now,

$$
2^{(2 \alpha-1) t_{1}} \leq 2^{(2 \alpha-1)\left(2+\log _{3}(2 / \epsilon)+\frac{2}{2 \alpha-1} \log _{3}\left(\frac{2 K_{1} d}{(2 \alpha-1) \theta}\right)\right)} \leq 2^{2+\log _{3}(2 / \epsilon)+\frac{2}{2 \alpha-1} \log _{3}\left(2 K_{1} /(2 \alpha-1)\right)} d^{\gamma}
$$

where

$$
\gamma=\frac{1}{2 \alpha+1} \frac{\ln 4}{\ln 3} \in\left(\frac{2}{5}, \frac{2}{3}\right)
$$

So, if

$$
K_{2}^{\prime \prime}=d^{-1 / 3} 2^{2+\log _{3}(2 / \epsilon)+\frac{2}{2 \alpha-1} \log _{3}\left(2 K_{1} /(2 \alpha-1)\right)}\left(\ln 4+\frac{2}{2 \alpha-1} \ln \left(K_{1} d /(2 \alpha-1)\right)\right),
$$


then

$$
\mathbf{E}(Z)<e^{-d^{2 / 3-\gamma}(2 /(2 \alpha-1)) \ln \left(K_{1} d /(2 \alpha-1)\right)} n / 4 \leq \epsilon_{1} n / 4 .
$$

It is straightforward to show that $Z$ is concentrated about its mean. A random pairing can be obtained by choosing a random permutation $\omega=\left(w_{1}, w_{2}, \ldots, w_{d n}\right)$ of $W$ and pairing $w_{2 i-1}$ with $w_{2 i}$, for $1 \leq i \leq d n / 2$. Interchanging two elements in $\omega$ can change $Z$ by at most two. Lemma 11 of [11] states: Let $S$ be a set with $|S|=N$. Let $\Omega$ be the set of $N$ ! permutations of $S$. Let $\omega$ be chosen uniformly from $\Omega$. Let $Z=Z(\omega)$ be such that $\left|Z(\omega)-Z\left(\omega^{\prime}\right)\right| \leq 1$ when $\omega^{\prime}$ is obtained from $\omega$ by interchanging two elements of the permutation.

$$
\operatorname{Pr}(|Z-\mathbf{E}(Z)| \geq t) \leq 2 e^{-2 t^{2} / N} .
$$

Using (8) with $N=d n$ and $t=(d n)^{1 / 2} \ln n$ yields the lemma.

We now appeal to a result of Alon, Krivelevich and Sudakov [4] to show that we can colour the graphs $\Gamma_{t}=G_{t}-B_{t}$ with few colours. The main result of that paper is

Theorem 2 There exists an absolute positive constant $C_{0}$ such that the following holds. Let $G$ be a graph with maximum degree $\Delta$ in which the neighbourhood of any vertex $v$ spans at most $\Delta^{2} / f$ edges. Then the chromatic number of $G$ is at most $C_{0} \frac{\Delta}{\ln f}$.

We use this to prove

Lemma 3 There exists $\beta>0$ such that

$$
\operatorname{Pr}\left(\exists t \in\left[0, t_{1}\right]: \chi\left(\Gamma_{t}\right) \geq \frac{C_{0} \Delta_{t}}{\beta \ln d}\right)=o(1) .
$$

Proof Fix $t$ and $v \in I_{t}$ and condition on the neighbours of $v$ in $G_{F}$ so that $v \notin B_{t}$. In so doing we fix at most $\Delta$ pairs of the configuration. With this conditioning the number of edges $\xi_{v}$ in the $\Gamma_{t}$-neighbourhood of $v$ is stochastically dominated by the Binomial $B\left(\Delta_{t}^{2} / 2, \Delta /(d n-2 \Delta)\right.$. So, if $\beta=4 / 3-2 \gamma>0(\gamma$ as in $(7))$ then

$$
\operatorname{Pr}\left(\xi_{v} \geq \frac{\Delta_{t}^{2}}{d^{\beta}}\right) \leq\left(\begin{array}{c}
\Delta_{t}^{2} / 2 \\
\Delta_{t}^{2} / d^{\beta}
\end{array}\right)\left(\frac{2 \Delta}{d n}\right)^{\Delta_{t}^{2} / d^{\beta}} \leq\left(\frac{e \Delta}{d^{1-\beta} n}\right)^{\Delta_{t}^{2} / d^{\beta}} .
$$

But $\Delta \leq n^{1 / 3}$ and $\Delta_{t}^{2} / d^{\beta} \geq 10$ for sufficiently large $K_{1}$ and so

$$
\operatorname{Pr}\left(\xi_{v} \geq \frac{\Delta_{t}^{2}}{d^{\beta}}\right)=o(1 / n)
$$

Applying Theorem 2 with $\Delta=\Delta_{t}$ and $f=d^{\beta}$ yields the lemma.

Lemma 4 If $F$ is chosen uniformly from the set $\Omega$ of pairings then whp

$$
\chi\left(G_{F}\right) \leq b_{1} \frac{d}{\ln d} .
$$


Proof Let $B_{t}, G_{t}, \Gamma_{t}, t \in\left[0, t_{1}\right]$, be as defined above. It follows from Lemma 3 that whp the number of colours needed to colour $\bigcup_{t=0}^{t_{1}} \Gamma_{t}$ is at most

$$
\sum_{t=0}^{t_{1}} \frac{C_{0} K_{2} d}{2^{(2 \alpha-1) t} \beta \ln d}=O\left(\frac{d}{\ln d}\right) \text {. }
$$

Finally note that the number of vertices in $B=\bigcup_{t=0}^{t_{1}} B_{t} \cup\left\{i \geq n-\frac{n}{3^{t_{1}+1}}\right\}$ is whp less than $\epsilon n$ and then, by Lemma 1 , the graph induced by $B$ is $\theta=d^{2 /(2 \alpha+1)}$-degenerate and so can be coloured with at most $\theta+1=O(d / \ln d)$ colours.

\subsection{From configurations to graphs}

It is at this point that we appeal to some results from Mckay and Wormald [15]. Where possible, we will use the terminology and notation of that paper. A loop of a pairing $F$ is a pair $\{u, v\}$ such that $\phi(u)=\phi(v)$. A double pair of $F$ is a pair $\left\{u_{1}, v_{1}\right\},\left\{u_{2}, v_{2}\right\} \in F$ such that $\phi\left(u_{1}\right)=\phi\left(u_{2}\right)$ and $\phi\left(v_{1}\right)=\phi\left(v_{2}\right)$. A double loop of $F$ is a pair of pairs $\left\{u_{1}, v_{1}\right\},\left\{u_{2}, v_{2}\right\}$ such that $\phi\left(u_{1}\right)=\phi\left(v_{1}\right)=\phi\left(u_{2}\right)=\phi\left(v_{2}\right)$. A triple pair is a triple of pairs $\left\{u_{i}, v_{i}\right\}, i=1,2,3$ such that $\phi\left(u_{1}\right)=\phi\left(u_{2}\right)=\phi\left(u_{3}\right)$ and $\phi\left(v_{1}\right)=\phi\left(v_{2}\right)=\phi\left(v_{3}\right)$.

In the lemmas that follow, we will assume always that Condition (b) of Theorem 1 holds.

Lemma 5 (Lemma 2 of [15])

The probability that $F$ contains at least one triple pair is $O\left(\Delta^{2} M_{2}^{2} / M_{1}^{3}\right)=o(1)$ and the probability of at least one double loop is $O\left(\Delta^{2} M_{2} / M_{1}^{2}\right)=o(1)$.

Let now $l$ denote the number of loops and $r$ denote the number of double pairs in $F$.

Lemma 6 (Lemma 3 of [15])

If $\lambda(n) \rightarrow \infty$ then whp

$$
l \leq 2 \Delta+\lambda \text { and } r \leq \Delta^{2}+\lambda
$$

We define the following two operations on a pairing: If $\phi(u)=i$ then we say that $u$ is in cell $i$.

I $l$-switching.

Take pairs $\left\{p_{1}, p_{6}\right\},\left\{p_{2}, p_{3}\right\},\left\{p_{4}, p_{5}\right\}$ where $\left\{p_{2}, p_{3}\right\}$ is a loop, and $p_{1}, \ldots, p_{6}$ are in five different cells. Replace these pairs by $\left\{p_{1}, p_{2}\right\},\left\{p_{3}, p_{4}\right\},\left\{p_{5}, p_{6}\right\}$. In this operation, none of the pairs created or destroyed is permitted to be part of a double pair. (See Figure 1). 


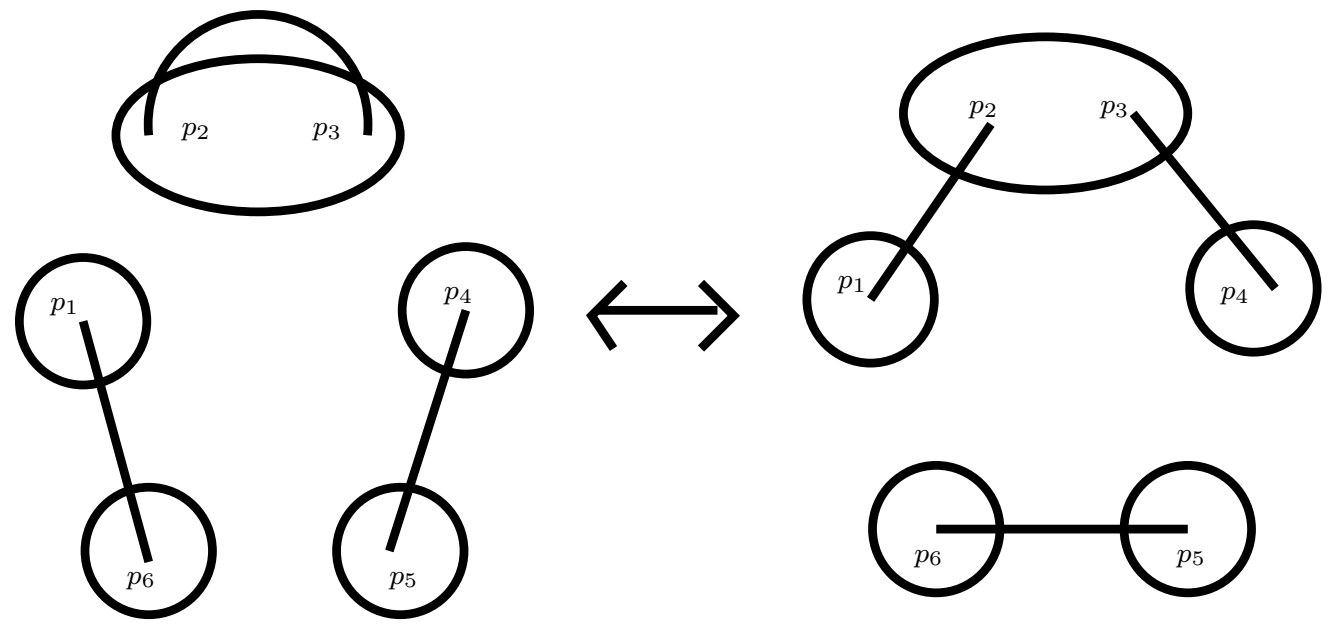

Figure 1:

II $r$-switching.

Take pairs $\left\{p_{1}, p_{5}\right\},\left\{p_{2}, p_{6}\right\},\left\{p_{3}, p_{7}\right\},\left\{p_{4}, p_{8}\right\}$ where $\phi\left(p_{2}\right)=\phi\left(p_{3}\right)$ and $\phi\left(p_{6}\right)=\phi\left(p_{7}\right)$, but the cells containing $p_{1}, p_{2}, p_{4}, p_{5}, p_{6}, p_{8}$ are all distinct. Replace these pairs by $\left\{p_{1}, p_{2}\right\},\left\{p_{3}, p_{4}\right\},\left\{p_{5}, p_{6}\right\},\left\{p_{7}, p_{8}\right\}$. In this operation, none of the pairs created or destroyed (other than the pairs $\left\{p_{2}, p_{6}\right\},\left\{p_{3}, p_{7}\right\}$ ) is permitted to be part of a multiple pair. (See Figure 2).

A forward $l$-switching is an $l$-switching as described, and a backward $l$-switching is the reverse operation. We use the same convention for $r$-switchings. Note that a forward $l$ switching always reduces the number of loops by one and does not create or destroy double pairs. Similarly, a forward $r$-switching reduces the number of double pairs by one and neither creates nor destroys loops.

Now let $\mathcal{C}_{l, r}$ denote the set of pairings $F$ with $l$ loops, $r$ double pairs and no triple pairs or double loops.

Lemma 7 (Lemma 4 of [15])

Denote an operation taking an element of $\mathcal{C}_{i, j}$ to an element $\mathcal{C}_{k, l}$ by $\mathcal{C}_{i, j} \rightarrow \mathcal{C}_{k, l}$. For each of the following operations, we bound the number, $m$, of ways of applying the operation to a fixed $F$. 

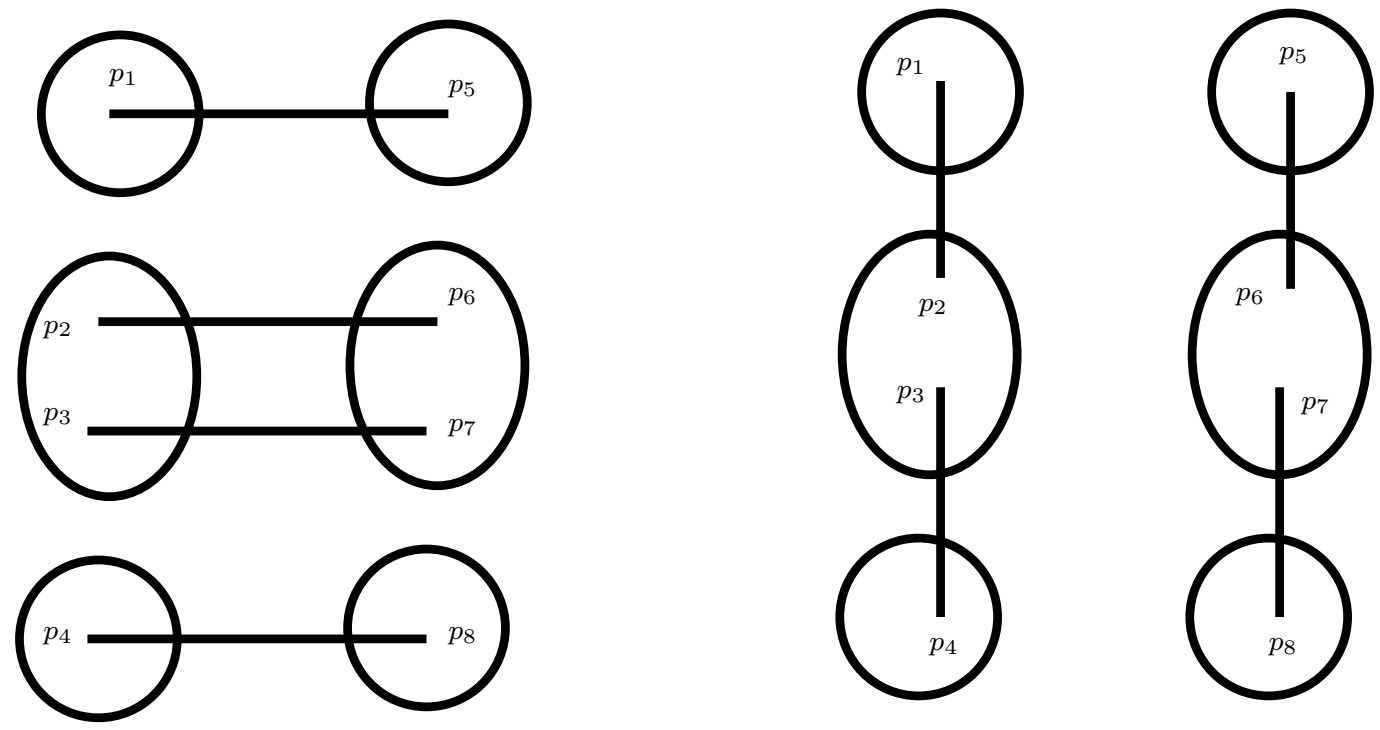

Figure 2:

(1) Forward l-switching $\mathcal{C}_{l, r} \rightarrow \mathcal{C}_{l-1, r}$ :

$$
2 l M_{1}^{2} \geq m \geq 2 l M_{1}^{2}\left(1-O\left(\frac{\Delta^{2}+l+r}{M_{1}}\right)\right) .
$$

(2) Backward l-switching $\mathcal{C}_{l-1, r} \rightarrow \mathcal{C}_{l, r}$ :

$$
M_{1} M_{2} \geq m \geq M_{1} M_{2}\left(1-\frac{\Delta(6(l+2 r)+\Delta l)}{M_{2}}-\frac{2 \Delta(\Delta+2)}{M_{1}}\right) .
$$

(3) Forward r-switching $\mathcal{C}_{0, r} \rightarrow \mathcal{C}_{0, r-1}$ :

$$
4 r M_{1}^{2} \geq m \geq 4 r M_{1}^{2}\left(1-O\left(\frac{\Delta^{2}+r}{M_{1}}\right)\right) .
$$

(4) Backward r-switching $\mathcal{C}_{0, r-1} \rightarrow \mathcal{C}_{0, r}$ :

$$
M_{2}^{2} \geq m \geq M_{2}^{2}\left(1-\frac{\Delta\left(16 r+9 \Delta+3+\Delta^{2}\right)}{M_{2}}\right) .
$$

Now consider the following algorithm for generating a member of $\mathcal{G}_{n, \mathbf{d}}$ :

1. Generate a random pairing $F$.

2. If there is a double loop or a triple pair, output $\perp-$ construed as failure. 
3. If the number of loops $l \geq 2 \Delta+\log n$ or the number of double pairs $r \geq \Delta^{2}+\log n$, output $\perp-$ construed as failure.

4. $F_{0} \leftarrow F$.

5. For $i=1$ to $l$ choose a random forward $l$-switching on $F_{i-1}$, creating $F_{i} \in \mathcal{C}_{l-i, r}$.

6. For $i=l+1$ to $l+r$ choose a random forward $r$-switching on $F_{i-1}$, creating $F_{i} \in$ $\mathcal{C}_{0, r-(i-l)}$.

7. Output $G^{*}=G_{F_{l+r}} \in \mathcal{G}_{n, \mathbf{d}}$.

For each $l, r$ satisfying (9), with $\lambda=\log n$, and $G \in \mathcal{G}_{n, \mathbf{d}}$, there are by Lemma $7(2),(4)$

$$
\left(M_{1} M_{2}\right)^{l} M_{2}^{r}\left(1+O\left(\frac{\Delta l(\Delta l+r)}{M_{2}}+\frac{\Delta^{2} l}{M_{1}}+\frac{\Delta r\left(\Delta^{2}+r\right)}{M_{2}}\right)\right)
$$

sequences of switchings which yield $G$. Each of these has probability

$$
\left(\left(2 M_{1}^{2}\right)^{l} l !\left(4 M_{1}^{2}\right)^{r} r !\right)^{-1}\left(1+O\left(\frac{l\left(\Delta^{2}+l+r\right)}{M_{1}}+\frac{r\left(\Delta^{2}+r\right)}{M_{1}}\right)\right)
$$

of being followed by the algorithm, given $l, r$.

Thus if Condition (b) holds, then whp the algorithm outputs a graph in $\mathcal{G}_{n, \mathbf{d}}$ and

$$
\operatorname{Pr}\left(G^{*}=G\right)=(1+o(1)) \sum_{l=0}^{2 \Delta+\log n} \sum_{r=0}^{\Delta^{2}+\log n} \frac{M_{1}^{l} M_{2}^{r+l}}{2^{l} M_{1}^{2(l+r)} l ! r !} \operatorname{Pr}(l \text { loops, } r \text { double pairs })
$$

and so for $G_{1}, G_{2} \in \mathcal{G}_{n, \mathbf{d}}$

$$
\operatorname{Pr}\left(G^{*}=G_{1}\right)=(1+o(1)) \operatorname{Pr}\left(G^{*}=G_{2}\right) .
$$

Given this, we only have to show that whp $\chi\left(G^{*}\right)=O\left(\chi\left(G_{F}\right)\right)$. Thus let $H$ be the graph induced by the edges that are added by the switchings. We will show that whp

$$
\Delta(H) \leq 5
$$

Since $\chi\left(G^{*}\right) \leq \chi(H) \chi\left(G_{F}\right) \leq(\Delta(H)+1) \chi\left(G_{F}\right)$, this will complete the proof of our upper bound.

Now every edge at distance $\geq 2$ from the loop or double edge can be used as one of the two edges destroyed by the two types of switching. Thus, vertex $i$ has probability

$$
O\left(\frac{d_{i}}{M_{1}-\Delta^{2}}\right)=O\left(\frac{\Delta}{M_{1}}\right)
$$


of having an $\mathrm{H}$-edge created in any switching, regardless of the history of the switchings to this point.

Thus, for some constant $c>0$, assuming due to Lemma 6 that $G^{*}$ satisfies (9):

$$
\operatorname{Pr}(\Delta(H) \geq 5) \leq n\left(\begin{array}{c}
\Delta^{2}+2 \Delta+2 \log n \\
5
\end{array}\right)\left(\frac{c \Delta}{M_{1}}\right)^{5} \leq n M_{1}^{5 / 2-15 / 4}=o(1)
$$

and this completes the proof of the upper bound in Theorem 1.

Note that we cannot immediately use the stronger result of [13]. This is because in that paper Lemma 7(4) is replaced by an average bound.

\section{Lower bound}

We will work with the configuration model.

We first observe that

$$
\operatorname{Pr}\left(G_{F} \text { is simple }\right) \geq e^{-\Delta^{2}} .
$$

This follows directly from the formula for the asymptotic number of labelled graphs with a degree sequence in which $\Delta=o\left(M_{1}^{1 / 4}\right)$, see [13].

Let

$$
t=b_{2} \frac{d}{\ln d},
$$

where $b_{2}>0$ is a sufficiently small constant. Fix a partition $\left(V_{1}, \ldots, V_{t}\right)$ of the vertex set of $G_{F}$ into $t$ parts. We will show:

$$
\operatorname{Pr}\left(\left(V_{1}, \ldots, V_{t}\right) \text { is a proper colouring of } G_{F}\right) \leq t^{-2 n} .
$$

This will be enough to beat both the union bound for the number of $t$-partitions (which is $\leq t^{n}$ ) and the inverse probability that $G_{F}$ is simple (which is $\leq e^{\Delta^{2}}-$ see (11)).

Observe first that

$$
\operatorname{Pr}\left(V_{i} \text { is independent in } G_{F}\right) \leq\left(1-\frac{D\left(V_{i}\right) / 2-\Delta}{d n}\right)^{D\left(V_{i}\right) / 2} .
$$

Explanation: Match the first $D\left(V_{i}\right) / 2$ points of $V_{i}$ in the configuration model. When matching a point, it should be matched either to a point belonging to a vertex outside of $V_{i}$, or to a point of the same vertex.

Now, if in the partition $\left(V_{1}, \ldots, V_{t}\right)$ one of the sets $V_{i}$ satisfies $D\left(V_{i}\right) \geq n d / 4$, by the above argument we get (taking $b_{2}$ small enough if necessary):

$$
\operatorname{Pr}\left(\left(V_{1}, \ldots, V_{t}\right) \text { is a } t \text {-colouring }\right) \leq \operatorname{Pr}\left(V_{i} \text { is independent }\right) \leq e^{-(1-o(1)) n d / 64} \ll t^{-2 n} \text {. }
$$


Assume therefore that $\left|V_{i}\right| \leq d n / 4$ for all $1 \leq i \leq t$. Assume w.l.o.g. that $D\left(V_{1}\right) \leq D\left(V_{2}\right) \leq$ $\cdots \leq D\left(V_{t}\right)$. Choose $1 \leq k \leq t$ so that

$$
\frac{d n}{4} \leq \sum_{i=1}^{k} D\left(V_{i}\right) \leq \frac{d n}{2}
$$

such a $k$ exists as $D\left(V_{i}\right) \leq n d / 4$ for all $i$.

We will estimate from above the probability that all of the sets $V_{i}, i=1, \ldots, k$, are independent. In order to do this, we will expose the pairings of the points of $V_{i}$, each time choosing a set $V_{i}$ that has at least half of its points unpaired, for as long as possible. Let $\sigma$ describe the order in which the sets $V_{1}, \ldots, V_{k}$ are exposed. Observe that

$\operatorname{Pr}\left(V_{\sigma(i)}\right.$ is independent $\mid V_{\sigma(1)}, \ldots, V_{\sigma(i-1)}$ are independent $)$

$$
\leq\left(1-\frac{D\left(V_{\sigma(i)}\right) / 4-\Delta}{d n}\right)^{D\left(V_{\sigma(i)}\right) / 4} \leq e^{-\frac{D\left(V_{\sigma(i)}\right)^{2}-4 \Delta D\left(V_{\sigma(i)}\right)}{16 d n}}
$$

(match the first half of yet unmatched points of $\left.V_{\sigma(i)}\right)$.

Suppose that we have in this way exposed the pairings of sets $V_{\sigma(1)}, \ldots, V_{\sigma(l)}$. If

$$
\sum_{i=1}^{l} D\left(V_{\sigma(i)}\right) \leq \frac{d n}{16}
$$

then at this moment the number of paired points does not exceed $d n / 8$, and therefore at least one of the unexposed sets $V_{i}, i \notin\{\sigma(1), \ldots, \sigma(l)\}$, has at least a half of its points unpaired, and we can proceed. Thus, if by the end of the process we have exposed pairings of sets $V_{\sigma(1)}, \ldots, V_{\sigma\left(l^{*}\right)}$, it follows that $\sum_{i=1}^{l^{*}} D\left(V_{\sigma(i)}\right) \geq d n / 16$ and therefore

$$
\sum_{i=1}^{l^{*}}\left(D\left(V_{\sigma(i)}\right)^{2}-4 \Delta D\left(V_{\sigma(i)}\right)\right) \geq \frac{(1-o(1)) d^{2} n^{2}}{256 l^{*}} \geq \frac{(1-o(1)) d^{2} n^{2}}{256 t} .
$$

Hence

$$
\begin{aligned}
& \operatorname{Pr}\left(\left(V_{\sigma(1)}, \ldots, V_{\sigma\left(l^{*}\right)} \text { are independent }\right)\right. \\
& \quad \leq \exp \left\{-\frac{\sum_{i=1}^{l^{*}}\left(D\left(V_{\sigma(i)}\right)^{2}-4 \Delta D\left(V_{\sigma(i))}\right)\right.}{16 d n}\right\} \leq \exp \left\{-\frac{d n}{4100 t}\right\} .
\end{aligned}
$$

Recalling the expression for $t$ and choosing the constant $b_{2}$ there to be small enough, we get the desired estimate.

This completes the proof of Theorem 1 .

Acknowledgement: We thank Paul Horn for pointing out an error in an earlier draft of the paper. 


\section{References}

[1] D. Achlioptas and C. Moore, The Chromatic Number of Random Regular Graphs, Proceedings of RANDOM 2004, 219-228.

[2] D. Achlioptas and A. Naor, The Two Possible Values of the Chromatic Number of a Random Graph, Annals of Mathematics 62 (2005), 1335-1351.

[3] W. Aiello, F. Chung and L. Lu. A random graph model for power law graphs, Experimental Mathematics 10, (2001), 53-66.

[4] N. Alon, M. Krivelevich and B. Sudakov, Coloring graphs with sparse neighborhoods, Journal of Combinatorial Theory, Series B 77 (1999), 73-82.

[5] E.A. Bender and E.R. Canfield, The asymptotic number of labelled graphs with given degree sequences, Journal of Combinatorial Theory, Series A 24 (1978) 296-307.

[6] B. Bollobás, A probabilistic proof of an asymptotic formula for the number of labelled regular graphs, European Journal on Combinatorics 1 (1980) 311-316.

[7] B. Bollobás, The chromatic number of random graphs, Combinatorica 8 (1988) 49-55.

[8] C. Cooper and A.M. Frieze, The size of the largest strongly connected component of a random digraph with a given degree sequence, Combinatorics, Probability and Computing 13 (2004) 319-338.

[9] C. Cooper, A.M. Frieze, B.A. Reed and O. Riordan, Random regular graphs of nonconstant degree: independence and chromatic number, Combinatorics, Probability and Computing 11 (2002) 323-342.

[10] A.M. Frieze and T. Euczak, On the independence and chromatic numbers of random regular graphs, Journal of Combinatorial Theory Series B 54 (1992) 123-132.

[11] A.M. Frieze and B.G.Pittel, Perfect matchings in random graphs with prescribed minimal degree, Trends in Mathematics, Birkhauser Verlag, Basel (2004) 95-132.

[12] T. Euczak, The chromatic number of random graphs, Combinatorica 11 (1991) 45-54.

[13] B.D. McKay and N.C. Wormald, Asymptotic enumeration by degree sequence of graphs with degree o( $\left.n^{1 / 2}\right)$, Combinatorica 11 (1991) 369-382.

[14] B.D.McKay and N.C.Wormald, Asymptotic enumeration by degree sequence of graphs of high degree, European Journal of Combinatorics 11 (1990) 565-580.

[15] B.D. McKay and N.C. Wormald, Uniform generation of random regular graphs of moderate degree, Journal of Algorithms 11 (1990) 52-67. 
[16] M. Molloy and B.A. Reed, A Critical Point for Random Graphs with a Given Degree Sequence, Random Structures and Algorithms 6 (1995) 161-180.

[17] M. Molloy and B.A. Reed, The Size of the Largest Component of a Random Graph on a Fixed Degree Sequence, Combinatorics, Probability and Computing 7 (1998) 295-306.

[18] N.C. Wormald Models of random regular graphs, Surveys in Combinatorics, London Mathematical Society Lecture Note Series 267, Cambridge University Press, Cambridge, 1999 (J.D.Lamb and D.A.Preece, Eds.), Proceedings of the 1999 British Combinatorial Conference, Cambridge University Press, 239-298. 